\title{
The specificity of interferon- $\gamma$-based blood tests in the identification of latent tuberculosis infection
}

\section{To the Editors:}

I have read with interest several papers describing the use of cellular interferon (IFN)- $\gamma$-based blood tests, including the editorial by DAVIES and DROBNIEWSKI [1]. Whilst reviewing the study by LEE et al. [2], DAVIES and DROBNIEWSKI [1] indicate that the T-SPOT.TB assay has higher sensitivity than QuantiFERON-TB Gold, but that the QuantiFERON-TB Gold may have better specificity.

I do not agree with this tentative conclusion as the study by LEE et al. [2] does not report the true specificity of the two tests, as the control group of 15- and 16-yr-old students used to examine the specificity of the assays, although healthy, had a defined risk of existing tuberculosis (TB) infection. This was acknowledged by LEE et al. [2] in the discussion section of their study as follows.

"The present study has several limitations. First, some of the low-risk subjects may actually have been infected with MTB [Mycobacterium tuberculosis] and this might lead to an underestimation of the specificity of the IFN- $\gamma$ assays. According to a South Korean national survey conducted in 1995, the MTB infection rate was $\sim 15 \%$ in $15 \mathrm{yr}$ olds, but rose dramatically to about $52-60 \%$ in 18-33 yr olds. Accordingly, 15-16-yr-old students were selected as a low-risk group because they have a lower chance of being infected with MTB."

T-SPOT.TB reported a $15.3 \%$ infection rate in this healthy control group versus $8.4 \%$ for QuantiFERON-TB Gold. In the absence of a true gold standard for latent TB infection (LTBI), it is impossible to definitely resolve whether the discrepancy between the T-SPOT.TB and QuantiFERON-TB Gold results in this group was due to the higher specificity of QuantiFERONTB Gold or the higher sensitivity of T-SPOT.TB. Nonetheless, the available data suggest that it is more likely that the discrepancy is due to the higher sensitivity of the T-SPOT.TB test rather than the higher specificity of QuantiFERON-TB Gold, for the following reasons.

First, LEE et al. [2] demonstrated that T-SPOT.TB has higher sensitivity than QuantiFERON-TB Gold in the diagnosis of culture-confirmed active TB disease, and this finding has also been reported by GOLETTI et al. [3]. Whilst active TB disease is clearly not the same as LTBI, it might be hypothesised that this difference in sensitivity is also true in LTBI. In the study by FERRARA et al. [4], this hypothesis was suggested by head-tohead evidence of the higher sensitivity of T-SPOT.TB over QuantiFERON-TB Gold in LTBI.

Secondly, both QuantiFERON-TB Gold and T-SPOT.TB use the same antigens (ESAT-6 and CFP-10). Therefore, it is hard to argue that T-SPOT.TB is any less specific for MTB infection than QuantiFERON-TB Gold, as both examine the cellular immune response to identical antigens. Furthermore, to my knowledge, there are no published data showing higher specificity for the QuantiFERON-TB Gold assay over the T-SPOT.TB assay. Additionally, four previous studies [5-8] investigating the specificity of the T-SPOT.TB test (using preapproval versions of the test) have demonstrated a specificity of $100 \%$, where truly low-risk controls in a low-endemicity country (UK) were studied.

Finally, Lee et al. [2] predicted the prevalence of tuberculosis infection in the healthy control cohort to be $15 \%$. The fact that T-SPOT.TB reported a rate of infection of $15.3 \%$ provides further evidence that T-SPOT.TB was detecting true latent tuberculosis infection. It is felt that, on the basis of the above arguments, the lower rate of detection by QuantiFERON-TB Gold is more likely to be attributed to the lower sensitivity of this assay, rather than its higher specificity.

\section{Granger}

Oxford Immunotec, 91 Milton Park, Abingdon, OX14 4RY, UK.

\section{STATEMENT OF INTEREST}

C. Granger is the director of professional relations of Oxford Immunotec, which manufactures the T-SPOT.TB assay.

\section{REFERENCES}

1 Davies PDO, Drobniewski F. The use of interferon- $\gamma$-based blood tests for the detection of latent tuberculosis infection. Eur Respir J 2006; 28: 1-3.

2 Lee JY, Choi HJ, Park I-N, et al. Comparison of two commercial interferon- $\gamma$ assays for diagnosing Mycobacterium tuberculosis infection. Eur Respir J 2006; 28: 24-30.

3 Goletti D, Carrara D, Vincenti D, et al. Accuracy of an immune diagnostic assay based on RD1 selected epitopes for active tuberculosis in a clinical setting: a pilot study. Clin Microbiol Infect 2006; 12: 544-550.

4 Ferrara G, Losi M, D'Amico R, et al. Use in routine clinical practice of two commercial blood tests for diagnosis of infection with Mycobacterium tuberculosis: a prospective study. Lancet 2006; 367: 1328-1334.

5 Lalvani A, Pathan AA, McShane H, et al. Rapid detection of Mycobacterium tuberculosis infection by enumeration of antigenspecific T cells. Am J Respir Crit Care Med 2001; 163: 824-828.

6 Pathan A, Wilkinson K, Klenerman P, et al. Direct ex vivo analysis of antigen-specific IFN- $\gamma$ secreting CD4 T cells in Mycobacterium tuberculosis-infected individuals: associations with clinical disease state and effect of treatment. J Immunol 2001; 167: 5217-5225.

7 Lalvani A, Nagvenkar P, Udwadia Z, et al. Enumeration of T cells specific for RD1-encoded antigens suggests a high prevalence of latent Mycobacterium tuberculosis infection in healthy urban Indians. J Infect Dis 2001; 183: 469-477.

8 Chapman A, Munkata M, Wilkinson K, et al. Rapid detection of active and latent tuberculosis infection in HIV-positive individuals by enumeration of Mycobacterium tuberculosisspecific T cells. AIDS 2002; 16: 2285-2293.

DOI: 10.1183/09031936.00107506 